\title{
CD36 identified by weighted gene co-expression network analysis as a hub candidate gene in lupus nephritis
}

\author{
Huiying Yang ${ }^{1}$, Hua Li ${ }^{\text {Corresp. } 1}$ \\ ${ }^{1}$ Department of Nephrology, Sir Run Run Shaw Hospital, Zhejiang University School of Medicine, Hangzhou, Zhejiang Province, China \\ Corresponding Author: Hua Li \\ Email address: h_li@zju.edu.cn
}

Lupus nephritis (LN) is a severe manifestation of systemic lupus erythematosus (SLE), which often progresses to end-stage renal disease (ESRD) and ultimately leads to death. At present, there are no definitive therapies towards $L N$, so that illuminating the molecular mechanism behind the disease has become an urgent task for researchers. Bioinformatics has become a widely utilized method for exploring genes related to disease. This study set out to conduct weighted gene co-expression network analysis (WGCNA) and screen the hub gene of LN. We performed WGCNA on the microarray expression profile dataset of GSE104948 from Gene Expression Omnibus (GEO) database with 18 normal and 21 LN samples of glomerulus. A total of 5,942 genes were divided into 5 co-expression modules, one of which was significantly correlated to LN. Gene Ontology (GO) and Kyoto Encyclopedia of Genes and Genomes (KEGG) enrichment analyses were conducted on the LN-related module and the module was proved to be associated mainly with the activation of inflammation, immune response, cytokines, and immune cells. Genes in the most significant GO terms were extracted for sub-networks of WGNCA. We evaluated the centrality of genes in the sub-networks by Maximal Clique Centrality (MCC) method and CD36 was ultimately screened out as a hub candidate gene of the pathogenesis of LN. The result was verified by its differentially expressed level between normal and LN in GSE104948 and the other three multi-microarray datasets of GEO. Moreover, we further demonstrated that the expression level of CD36 is related to the WHO Lupus Nephritis Class of LN patients with the help of Nephroseq database. The current study proposed $C D 36$ as a vital candidate gene in LN for the first time and CD36 may perform as a brandnew biomarker or therapeutic target of $L N$ in the future. 
2 CD36 identified by weighted gene co-expression

3 network analysis as a hub candidate gene in lupus

4 nephritis

5

6

7

8

9

10

11

12

13

14

15

16

17

Huiying Yang ${ }^{1}, \mathrm{Hua} \mathrm{Li}^{1}$

${ }^{1}$ Department of Nephrology, Sir Run Run Shaw Hospital, Zhejiang University School of Medicine, Hangzhou, China

Corresponding Author:

Hua Li1

3 Qingchun East Road, Hangzhou, Zhejiang province, China

Email address: h_li@zju.edu.cn

\section{Abstract}

Lupus nephritis (LN) is a severe manifestation of systemic lupus erythematosus (SLE), which often progresses to end-stage renal disease (ESRD) and ultimately leads to death. At present, there are no definitive therapies towards LN, so that illuminating the molecular mechanism behind the disease has become an urgent task for researchers. Bioinformatics has become a widely utilized method for exploring genes related to disease. This study set out to conduct weighted gene co-expression network analysis (WGCNA) and screen the hub gene of LN. We performed WGCNA on the microarray expression profile dataset of GSE104948 from Gene Expression Omnibus (GEO) database with 18 normal and $21 \mathrm{LN}$ samples of glomerulus. A total of 5,942 genes were divided into 5 co-expression modules, one of which was significantly correlated to LN. Gene Ontology (GO) and Kyoto Encyclopedia of Genes and Genomes (KEGG) enrichment analyses were conducted on the LN-related module and the module was proved to be associated mainly with the activation of inflammation, immune response, cytokines, and immune cells. Genes in the most significant GO terms were extracted for sub-networks of WGNCA. We evaluated the centrality of genes in the sub-networks by Maximal Clique Centrality (MCC) method and CD36 was ultimately screened out as a hub candidate gene of the pathogenesis of LN. The result was verified by its differentially expressed level between normal and LN in GSE104948 and the other three multi-microarray datasets of GEO. Moreover, we further demonstrated that the expression level of CD36 is related to the WHO Lupus Nephritis Class of LN patients with the help of Nephroseq database. The current study proposed CD36 as a vital candidate gene in $\mathrm{LN}$ for the first time and CD36 may perform as a brand-new biomarker or therapeutic target of $\mathrm{LN}$ in the future. 
39

40

41

42

43

44

45

46

47

48

49

50

51

52

53

54

55

56

57

58

59

60

61

62

63

64

65

66

67

68

69

70

71

72

73

74

75

76

77

78

\section{Introduction}

Systemic lupus erythematosus (SLE) is a chronic, systemic autoimmune disease characterized by autoantibody production, complement activation and immune complex deposition. The incidence of SLE ranges from $0.03 \%$ to $2.32 \%$ person-years worldwide (Rees et al. 2017).

Lupus nephritis (LN) is one of the most frequent and severe organ manifestations in patients with SLE, the hallmark of which is often glomerulonephritis. Approximately $50 \%$ of SLE patients develop clinically evident renal disease, up to $11 \%$ of whom develop end-stage renal disease (ESRD) at 5 years (Tektonidou, Dasgupta and Ward 2016, Almaani, Meara and Rovin 2017). LN is an important cause of ESRD and mortality. The initial and subsequent therapy of LN mainly consists of immunosuppressants and glucocorticoids, which means there are little efficient and specific therapies. Thus, it is a pressing task to clarify molecular mechanisms involved in LN. $\mathrm{LN}$ is characterized by its complicated physiopathologic mechanism. In LN patients, the formation of immune complexes in different glomerular compartments, the activation of innate immune signal pathways, the infiltration of immune cells and proinflammatory mediators can harm glomerular cells through various approaches (Devarapu et al. 2017). Although many studies have determined certain pathological mechanisms of LN, the pathogenesis is still far from clear.

With the development of gene microarray and high-throughput next-generation sequencing, bioinformatics analysis of gene expression profiling has been broadly applied to explore the mechanism underlying diseases and potential diagnostic biomarkers or treatment targets. Among diverse means aiming to investigate altered molecular elements based on comparison between groups of different states, weighted gene co-expression network analysis (WGCNA) is a powerful tool utilized for describing the correlation patterns among genes and exploring hub genes related to certain traits (van Dam et al. 2018, Langfelder and Horvath 2008). WGCNA constructs a co-expression network between genes, and then, genes are divided into several coexpression modules by clustering techniques. Genes in certain module are deemed to share similar biological function and biological process. At last, after relating modules to clinical traits, modules with high correlation to disease are further analyzed and hub genes of pivotal importance to disease are identified. WGCNA has been broadly used for studying of diseases such as cancer (Jardim-Perassi et al. 2019), neuropsychiatric disorder (Huggett and Stallings 2019), chronic disease (Morrow et al. 2015) and proved to be quite useful.

However, although researchers have conducted numerous bioinformatics studies about LN and have got many achievements (Arazi et al. 2019, Panousis et al. 2019), WGCNA has rarely been used for studies of LN (Sun et al. 2019). In our study, we constructed a co-expression network of the expression profile of glomerulus tissue by WGCNA and confirmed gene modules related to LN. After systematically analyzing the LN-related co-expression module by series of bioinformatics methods, a hub gene associated with LN was identified and verified. Depend on the potential roles of the hub gene in the pathogenesis of LN, we expect to propose novel clues of the diagnosis and treatment of $\mathrm{LN}$.

Materials \& Methods 


\section{Expression profile data collection}

80 The overall procedures of our study are illustrated in the flow chart (Fig. 1). The gene expression 81 profile of GSE104948 was selected and obtained from the Gene Expression Omnibus (GEO)

82 database (https://www.ncbi.nlm.nih.gov/geo/). The raw data is available in the GEO database.

83 The dataset consists of microarray-based gene expression profiles of $32 \mathrm{LN}$ samples and 18

84 normal samples of glomerular tissues from SLE patients and living kidney transplant donors

85 respectively. The glomerular tissues were microdissected and verified with glomerular-selective

86 transcripts (Grayson et al. 2018). The expression values have already been log2 transformed.

87 Data preprocessing

88 The probe annotation was conducted under the Perl environment with the microarray platform

89 file. Probes matching with multiple genes were removed, and for genes corresponding to

90 multiple probes, the average values of probes were regarded as the expression values of the

91 genes. A total of 11,884 genes were left after the probe annotation.

92 Since non-varying genes are usually regarded as background noise, we filtered the genes by

93 variance and the top 50\% (5,942 genes) with larger variance were chosen for subsequent

94 analyses.

95 Weighted co-expression network construction and module division

96 Before WGCNA, we excluded the outlier samples by sample clustering with the hierarchical

97 clustering method. The sample IDs and details of all samples included in our study are available

98 in Table. S1. After that, we applied WGCNA with the expression profile by using the WGCNA

99 package (Langfelder and Horvath 2008) in the R environment (version 3.5.3). Firstly, we

100 calculated the Pearson's correlation for all pair-wise genes and constructed a correlation matrix.

101 Secondly, the correlation matrix was transformed to an adjacency matrix (also known as scale-

102 free network) with an appropriate soft-thresholding value $\beta$. A reasonable $\beta$ value would

103 emphasize strong correlations between genes and penalize weak ones. We calculated the scale-

104 free fit index and mean connectivity of each $\beta$ value from 1 to 30 respectively, and when the

105 scale-free fit index is up to 0.85 , the $\beta$ value with highest mean connectivity is deemed as the

106 most appropriate one. Then, the adjacency matrix was converted to a topological overlap matrix

107 (TOM) so that the indirect correlations between genes are concerned. Finally, we used average

108 linkage hierarchical clustering according to the TOM-based dissimilarity measure to classify all

109 genes into several co-expression modules with a minimum size of 30 genes, thereby the genes

110 with similar expression patterns were divided into the same module. After defining the first

111 principal component of a given module as eigengene, we calculated the Pearson's correlations of

112 the eigengenes, and merged modules whose eigengenes were highly correlated (with Pearson`s

113 correlation higher than 0.75 ) into one module.

114 To verify the reliability of the division of modules, we plotted an adjacency heatmap of all the

1155,942 genes analyzed by WGCNA. Besides, we completed a cluster analysis of module

116 eigengenes and plotted an adjacency heatmap to find out the interactions among modules.

117 Identification of clinically significant modules 
118 The clinical traits of our samples included normal and LN, we calculated the correlation between 119 modules and traits. Modules of positive correlation with LN were considered as playing roles in 120 the pathogenesis of the disease. On the other hand, genes in modules of positive correlation with

121

122

123

124

125

126

127

128

129

130

131

132

133

134

135

136

137

138

139

140

141

142

143

144

145

146

147

148

149

150

151

152

153

154

155

156

157

normal trait are indispensable for maintaining normal biological functions. Thus, we extracted gene modules of highest correlation with LN and normal for subsequent studies.

Here, we introduced the definition of gene significance (GS) and module membership (MM), which represent the correlation of a given gene with clinical trait and module eigengene respectively. Genes in clinical-related modules should have high values and preferable correlations of GS and MM.

GO and KEGG pathway enrichment analyses

To explore the involved signal pathways and biological characteristics of genes in clinicalrelated modules, we conducted Gene Ontology (GO) enrichment analysis and Kyoto Encyclopedia of Genes and Genomes (KEGG) pathway enrichment analysis and visualized the top 10 significant terms respectively with the clusterProfiler $\mathrm{R}$ package (Yu et al. 2012). For both of GO and KEGG, enrichment terms arrived the cut-off criterion of $p$-value $<0.01$ and Benjamin-Hochberg adjusted $p$-value $<0.01$ were considered as significant ones.

\section{Differentially expressed genes analysis}

To investigate the difference of the expression profiles between normal and LN samples of genes in clinical-related modules, we applied differentially expressed genes (DEGs) analysis based on Empirical Bayes test with the limma R package (Ritchie et al. 2015). The cut-off criterion was set as follow: $\mid \log 2$ fold change $(\log F C) \mid>1 ; p$-value $<0.01$; false discovery rate $(F D R)<0.001$. The results were visualized with the heatmap R package (Galili et al. 2018).

\section{Identification of hub gene}

Hub gene of the LN-related module should have high connectivity with the whole module and LN trait, which may play critical roles in the molecular mechanism of LN. For identifying the hub gene related with LN, we extracted gene clusters that enriched in certain GO terms from the WGCNA network to construct sub-networks after GO enrichment analysis of the LN-related module. Then, we utilized the Cytoscape software and its plug-in cytohubba to seek out the hub gene from sub-networks (Shannon et al. 2003). After calculating the Maximal Clique Centrality (MCC) value of each gene, those with high MCC values was regarded as hub genes (Chin et al. 2014). The results were exhibited with the Cytoscape software. We then surveyed the GS value, MM value and $\log F C$ value of the selected hub gene to validate its reasonability.

\section{Validation of hub gene with the other GEO datasets}

To further verify the differential expression level of the hub gene between normal and LN tissues, we analyzed the $\operatorname{logFC}$ value of the hub gene with data from the other three GEO datasets (GSE32591, GSE99339 and GSE113342). The GEO IDs and details of the datasets were given in Table. S1.

\section{Validation of the clinical significance of hub gene by Nephroseq database}

To assess the relationship between the expression level of the hub gene and the activity or grade of LN, we visited the Nephroseq database (http://v5.nephroseq.org), which provides unique 
158

159

160

161

162

163

164

165

166

167

168

169

170

171

172

173

174

175

176

177

178

179

180

181

182

183

184

185

186

187

188

189

190

191

192

193

194

195

196

access to datasets from the Applied Systems Biology Core at the University of Michigan, incorporating clinical data which is often difficult to collect from public sources. We then analyzed the difference of the expression level of hub gene between patients in different WHO Lupus Nephritis Class (Weening et al. 2004) based on two datasets (the dataset of Peterson Lupus Glom and the dataset of Berthier Lupus Glom) from Nephroseq database (details available in Supplemental Document. 1). We performed unpaired $t$ test for comparisons between groups and set the criterion of two-tailed value of $p<0.05$ as statistically significant.

\section{Results}

\section{Data preprocessing}

After data preprocessing, 5,942 genes were selected for subsequent analyses. Sample clustering excluded the outlier samples and a total of 18 normal samples and $21 \mathrm{LN}$ samples were left. The final result of sample clustering is shown in Fig. 2A, revealing satisfactory intra-group consistency and distinct difference between groups. The GEO IDs and details about the source of the samples are available in Table. S1.

\section{Weighted co-expression network construction and module division}

In the current study, taking both scale-free fit index and mean connectivity as reference, the softthresholding was determined as 10 (Fig. 2B and Fig. 2C). Accordingly, the correlation matrix was transformed to an adjacency matrix and then converted to a topological overlap matrix. Based on average linkage hierarchical clustering and module merging, the genes were divided into 6 modules and were displayed with different colors (Fig. 3A), including the black, blue, brown, magenta, pink, and grey modules, containing 220, 2,881, 777, 465, 770, and 829 genes, respectively. Genes in the grey module were those couldn't be divided into any co-expression modules.

Fig. 3B depicts the topological overlap adjacency among all the 5,942 genes analyzed by WGCNA, indicating that most genes have higher correlation with genes in the same module and lower correlation with genes in other modules, which means the division of the modules was accurate. The clustering dendrogram and adjacency heatmap of eigengene are shown in Fig. 3C and Fig. 3D, meaning that the 5 modules were mainly separated into two clusters.

\section{Identification of clinically significant modules}

We calculated the module-trait correlation coefficients and showed the results in Fig. 4A. The results illuminated that the blue module displayed highest correlation with $\mathrm{LN}$ trait $(\mathrm{r}=0.91, p=$ $2 \mathrm{e}-15)$, while the brown module related best with normal trait $(\mathrm{r}=0.66, p=4 \mathrm{e}-06)$. The GS and MM value of all member genes of the blue and brown modules were shown in the scatterplots (Fig. 4B and Fig. 4C). The GS and MM value were of high correlation in the two modules (cor = $0.89, p<1 \mathrm{e}-200$, and cor $=0.73, p=3.1 \mathrm{e}-130$ respectively), suggesting that the genes in the two modules were associate with their module eigengenes and clinical traits synchronously and thus suitable for further analyses and hub gene excavation. We then renamed the two modules as top LN module and top non-LN module respectively.

\section{DEGs analysis of trait-related modules}


197 We applied DEGs analysis for the two trait-related modules. For the top LN module, 203 DEGs

198

199

200

201

202

203

204

205

206

207

208

209

210

211

212

213

214

215

216

217

218

219

220

221

222

223

224

225

226

227

228

229

230

231

232

233

234

235

236 were screened in LN compare with normal, including 195 up-regulated genes and 8 downregulated ones. While, the top non-LN module contained 78 DEGs, all of which were downregulated. The top 30 of up-regulated and down-regulated genes are displayed in Fig. 5 respectively. The $\log F C$ value, $p$-value, and FDR of DEGs are given in Table. S2.

\section{GO and KEGG Enrichment analyses of trait-related modules}

To confirm the biological themes of genes in the trait-related modules and find the underlying biological pathways behind LN, we performed GO and KEGG enrichment analyses towards the top LN and top non-LN modules and the top 10 significant terms of GO and KEGG are exhibited in Fig. 6 and Fig. 7 respectively. The complete results of GO and KEGG enrichment analyses were given in Table. S3.

For the top LN module, enriched GO-BP terms were mainly about activation of immune response, immune cells, cytokine production, and inflammation (Fig. 6A), such as "neutrophil activation" (gene count $=178, p=7.26 \mathrm{e}-301.52 \mathrm{E}-20)$, "positive regulation of defense response" (gene count $=160, p=9.14 \mathrm{e}-25)$, "activation of innate immune response" $($ gene count $=107, p=$ 3.45e-18). Enriched GO-MF terms were mainly about cytokine and their receptors (Fig. 6B), such as "cytokine binding" (gene count $=39, p=2.47 \mathrm{e}-8$ ), "cytokine receptor activity" (gene count $=34, p=5.3 \mathrm{e}-7)$, "cytokine receptor binding" (gene count $=69, \mathrm{p}=2.98 \mathrm{e}-5)$. For GO-CC, enriched terms were mainly involved in membrane and endocytic vesicle (Fig. 6C), such as "vesicle lumen" (gene count $=107, p=4.13 \mathrm{e}-15$ ), "cytoplasmic vesicle lumen" (gene count = $106, p=8.99 \mathrm{e}-15)$, "membrane region" (gene count $=95, p=2.33 \mathrm{e}-12$ ). The results of KEGG enrichment were similar to that of GO-BP, were mainly about immune and inflammation (Fig. 7A). The top KEGG terms included "Complement and coagulation cascades" (gene count $=31, p$ $=6.55 \mathrm{e}-6$ ), "Fc gamma R-mediated phagocytosis" (gene count $=34, p=1.90 \mathrm{e}-5$ ), "Th1 and Th2 cell differentiation" (gene count $=33, p=3.05 \mathrm{e}-5$ ). Meanwhile, several well-known pathways involved in LN were also included, such as Th17 cell differentiation.

On the other hand, for the top non-LN module, the GO-BP results were mainly involved in the metabolism process of different kinds of molecule (Fig. 6D), for example, "small molecule catabolic process" (gene count $=93, p=3.27 \mathrm{e}-41$ ), "organic acid catabolic process" (gene count $=73, p=3.97 \mathrm{e}-39)$, "carboxylic acid catabolic process" (gene count $=73, p=3.97 \mathrm{e}-39$ ). The top 10 terms of GO-MF and GO-CC are also displayed (Fig. 6E and Fig. 6F). Similarly, the top KEGG terms were mainly about metabolism process (Fig. 7B).

Besides, we found that the magenta module also had high correlation with LN trait. The results of enrichment analyses of magenta module are also given in Table. S3.

\section{Identification and validation of hub gene}

We extracted the genes enriched in two of the top 10 significant GO-BP terms in top LN module, namely "neutrophil activation" and "positive regulation of defense response", and constructed two sub-networks of the weighted co-expression network respectively. Co-expression pairs with top 500 weighted correlations in the sub-networks were selected for hub gene excavation. After importing the gene co-expression pairs and their weighted correlations into Cytoscape, we

Peer) reviewing PDF | (2019:07:39216:2:0:NEW 20 Aug 2019) 
237 calculated the MCC value of genes and the most central genes of the sub-network were screened 238 out as shown in Fig. 8. In both sub-networks, CD36 had the maximum MCC value among all, 239 and was therefore deemed as the hub gene under the pathogenesis of LN.

240 The GS and MM value of $C D 36$ were 0.772 and 0.833 , revealing $C D 36$ was significant 241 correlated with the top LN module and LN trait. The DEGs analysis showed that the expression

242 level of CD36 in LN was abnormally up-regulated compared with that of normal. The $\operatorname{logFC}$ of 243 CD36 was 2.298, which ranked 11th among all.

\section{Validation of hub gene with the other GEO datasets}

245 For verifying our conclusion in a broader range, we interrogated the GEO database for more

246 datasets about LN. We downloaded the datasets of GSE32591, GSE99339 and GSE113342, and

247 then analyzed the differentially expressed level of CD36 between LN and normal (Fig. 9). In the

248 four different datasets, the expression level of CD36 was consistently up-regulated in LN

249 samples, illustrating a satisfactory reliability of the result.

250 Validation of hub gene by Nephroseq database

251 We analyzed the expression level of CD36 in glomerular tissues under different severity of LN

252 evaluated by WHO Lupus Nephritis Class. The result indicates that $C D 36$ was significantly up253 regulated with the aggravation of $\mathrm{LN}$ (Fig. 10), that is to say, CD36 plays an important role in 254 the development of LN.

\section{Discussion}

256 In the current study, we used the expression profile of GSE104948 to screen the hub gene 257 involved in the pathogenesis of LN. We performed WGCNA and divided all genes into 5 co258 expression modules. After relating the modules to clinical traits, we concluded that the blue 259 module was of highest correlation with LN and was suitable for hub gene excavating. The brown 260 module had the highest correlation with normal trait, and was also worthy of subsequent

261

262

263

264

265

266

267

268

269

270

271

272

273

274

275 analyses. GO and KEGG enrichment illuminated that genes in the top LN module were mostly enriched in biological themes of the activation of inflammation, immune response, cytokine, and immune cells, and the top non-LN module was mainly about the metabolism process of various molecules. What's more, DEGs analysis showed that almost all DEGs in top LN module were abnormally up-regulated, revealing an aberrant activated stage of inflammation and immune response in LN. On the other hand, all DEGs in the top non-LN module were down-regulated, meaning a reduced ability of material metabolism in LN. To achieve the ultimately purpose of finding out the hub gene, we extracted genes enriched in the GO terms of "neutrophil activation" and "positive regulation of defense response" and constructed sub-networks accordingly. Base on the MCC method, $C D 36$ with maximum value of MCC in both sub-networks was regarded as the hub gene behind the pathogenesis of LN. We investigated the GS, MM and $\log F C$ of $C D 36$ and validated its importance. We interrogated the GEO database and got more datasets of LN, the obvious overexpression of $C D 36$ was therefore further verified. Moreover, the association between CD36 and WHO Lupus Nephritis Class showed directly that the expression level of CD36 gradually up-regulates along with the development of $\mathrm{LN}$ evaluated by WHO Lupus 
276 Nephritis Class, providing strong evidence that the abnormal over-expression of $C D 36$ is an 277 important element in the pathogenesis of LN.

278 The CD36 gene is located on band q11.2 of chromosome 7 (Fernandez-Ruiz et al. 1993). The

279 protein encoded by $C D 36$ is a kind of transmembrane protein (also known as scavenger receptor 280 B2) expresses on the surface of various kinds of cells. In the glomerulus of kidney, CD36 281 expresses in podocyte (Hua et al. 2015), mesangial cells (Ruan et al. 1999) and interstitial 282 macrophages (Kennedy et al. 2013). Meanwhile, CD36 expresses in immunity correlating cells 283 such as monocytes and macrophages (Collot-Teixeira et al. 2007). With multiple ligands, CD36

284

285

286

287

288

289

290

291

292

293

294

295

296

297

298

299

300

301

302

303

304

305

306

307

308

309

310

311

312

313

314 involves in complex biological process such as lipid homeostasis, immune response and cell apoptosis.

There are no relative reports concerning the direct relationship between $C D 36$ and $\mathrm{LN}$. Our results have shown that $C D 36$ mainly participates in the function of neutrophil, such as "neutrophil activation", "neutrophil activation involved in immune response", "neutrophil degranulation", "neutrophil mediated immunity", as well as the activation of immune response, such as "positive regulation of defense response", "positive regulation of innate immune response", "innate immune response-activating signal transduction", "positive regulation of immune effector process". Base on the results, we conclude that CD36 performs important functions in the pathogenesis and development of LN through affecting the function of neutrophil and innate immune response. Studies have proved that deposited immune complexes (IC) could activate complement and attract neutrophils and potentiate their responses, which will lead to intense glomerulonephritis, release protease and Reactive Oxygen Species (ROS), and give rise to kidney involvement of SLE (Tsuboi et al. 2008). Besides, IC-induced activation of neutrophils can lead to the formation of neutrophil extracellular traps (NETs), which can be pathogenic and promote the release of type I interferon (Garcia-Romo et al. 2011). CD36 may candidate in the pathogenesis of LN by the above-mentioned pathways. Numerous studies have shown that $C D 36$ participates in the pathogenesis of several kinds of chronic kidney disease (CKD).

It has long been known that chronic inflammation is an important segment of the progression of CKD. Studies have proved that the ligands signal via $C D 36$ to promote inflammatory response and the recruitment and activation of macrophage in the glomerulus (Kennedy et al. 2013). A report of LN showed that renal macrophage is associated with onset of nephritis and indicates poor prognosis (Bethunaickan et al. 2011). Meanwhile, oxidant stress plays a critical role in glomerular dysfunction. Along with chronic inflammation, $C D 36$ may facilitate the development of oxidant stress in LN (Hua et al. 2015, Kennedy et al. 2013, Aliou et al. 2016).

Podocyte is most susceptible to injury among the component of the glomerulus and its injury leads to glomerular dysfunction in various renal diseases including LN. Here, we determined that podocyte functional markers were down-ragulated in LN glomerular tissue, including WT1 $(\operatorname{logFC}=-0.273, p=0.009)$ and NPHS1 $(\log \mathrm{FC}=-0.306, p=0.034)$, indicating the fact of podocyte injury. It is reported that in primary nephrotic syndrome mouse, the overexpression of 
315 CD36 in the podocyte promotes its apoptosis (Yang et al. 2018). There is probably similar 316 pathogenesis in the progression of LN.

317 Ectopic lipid deposition in kidney may cause lipotoxicity and further affect the function of the 318 kidney (Lin et al. 2019). CD36 is a multifunctional protein function as a key molecule in the 319 uptake of long-chain fatty acids, which is the main component of fatty acids uptake system in the 320 kidney and plays a critical rule in the development of CKD (Gai et al. 2019). The expression 321 level of $C D 36$ is higher in kidney with acute or chronic damage, and lipid disorders will 322 stimulate the up-regulation of $C D 36$. Furthermore, $C D 36$ can promote the uptake of lipid from

323

324

325

326

327

328

329

330

331

332

333

334

335

336

337

338

339

340

341

342

343

344

345

346

347

348

349

350

351

352

353 plasma to tissue (Hua et al. 2015, Lin et al. 2019, Nosadini and Tonolo 2011, Yang et al. 2017). Among our results, the top $10 \mathrm{GO}$ terms of non-LN module includes two terms about lipid metabolism: fatty acid catabolic process $(p=8.03 \mathrm{e}-22)$ and fatty acid metabolic process $(p=$ 2.62e-20), implying that lipid disorders exist in the glomerular tissue, in which CD36 may take part.

Regrettably, as most reports on the relationship between $C D 36$ and kidney diseases are about the disorders of kidney in metabolic diseases, there is no study about the role $C D 36$ plays in immune response, which is worthy of further study in LN.

\section{Conclusions}

In conclusion, through WGCNA and a series of comprehensive bioinformatics analyses, $C D 36$ was confirmed for the first time as a hub gene in the pathogenesis of LN. CD36 is likely to become a new biomarker or therapeutic target of LN. Our work might provide a new insight for exploring the molecular mechanisms of LN.

\section{References}

Aliou, Y., M. C. Liao, X. P. Zhao, S. Y. Chang, I. Chenier, J. R. Ingelfinger \& S. L. Zhang (2016) Post-weaning high-fat diet accelerates kidney injury, but not hypertension programmed by maternal diabetes. Pediatr Res, 79, 416-24.

Arazi, A., D. A. Rao, C. C. Berthier, A. Davidson, Y. Liu, P. J. Hoover, A. Chicoine, T. M. Eisenhaure, A. H. Jonsson, S. Li, D. J. Lieb, F. Zhang, K. Slowikowski, E. P. Browne, A. Noma, D. Sutherby, S. Steelman, D. E. Smilek, P. Tosta, W. Apruzzese, E. Massarotti, M. Dall'Era, M. Park, D. L. Kamen, R. A. Furie, F. Payan-Schober, W. F. Pendergraft, 3rd, E. A. McInnis, J. P. Buyon, M. A. Petri, C. Putterman, K. C. Kalunian, E. S. Woodle, J. A. Lederer, D. A. Hildeman, C. Nusbaum, S. Raychaudhuri, M. Kretzler, J. H. Anolik, M. B. Brenner, D. Wofsy, N. Hacohen \& B. Diamond (2019) The immune cell landscape in kidneys of patients with lupus nephritis. Nat Immunol, 20, 902-914.

Almaani, S., A. Meara \& B. H. Rovin (2017) Update on Lupus Nephritis. Clin J Am Soc Nephrol, 12, 825-835.

Bethunaickan, R., C. C. Berthier, M. Ramanujam, R. Sahu, W. Zhang, Y. Sun, E. P. Bottinger, L. Ivashkiv, M. Kretzler \& A. Davidson (2011) A unique hybrid renal mononuclear phagocyte activation phenotype in murine systemic lupus erythematosus nephritis. J Immunol, 186, 49945003 . 
354 Chin, C. H., S. H. Chen, H. H. Wu, C. W. Ho, M. T. Ko \& C. Y. Lin (2014) cytoHubba:

355

356

357

358

359

360

361

362

363

364

365

366

367

368

369

370

371

372

373

374

375

376

377

378

379

380

381

382

383

384

385

386

387

388

389

390

391

392

identifying hub objects and sub-networks from complex interactome. BMC Syst Biol, 8 Suppl 4, S11.

Collot-Teixeira, S., J. Martin, C. McDermott-Roe, R. Poston \& J. L. McGregor (2007) CD36 and macrophages in atherosclerosis. Cardiovasc Res, 75, 468-77.

Devarapu, S. K., G. Lorenz, O. P. Kulkarni, H. J. Anders \& S. R. Mulay (2017) Cellular and Molecular Mechanisms of Autoimmunity and Lupus Nephritis. Int Rev Cell Mol Biol, 332, 43154.

Fernandez-Ruiz, E., A. L. Armesilla, F. Sanchez-Madrid \& M. A. Vega (1993) Gene encoding the collagen type I and thrombospondin receptor CD36 is located on chromosome 7q11.2. Genomics, 17, 759-61.

Gai, Z., T. Wang, M. Visentin, G. A. Kullak-Ublick, X. Fu \& Z. Wang (2019) Lipid Accumulation and Chronic Kidney Disease. Nutrients, 11.

Galili, T., A. O'Callaghan, J. Sidi \& C. Sievert (2018) heatmaply: an R package for creating interactive cluster heatmaps for online publishing. Bioinformatics, 34, 1600-1602.

Garcia-Romo, G. S., S. Caielli, B. Vega, J. Connolly, F. Allantaz, Z. Xu, M. Punaro, J. Baisch, C. Guiducci, R. L. Coffman, F. J. Barrat, J. Banchereau \& V. Pascual (2011) Netting neutrophils are major inducers of type I IFN production in pediatric systemic lupus erythematosus. Sci Transl Med, 3, 73ra20

Grayson, P. C., S. Eddy, J. N. Taroni, Y. L. Lightfoot, L. Mariani, H. Parikh, M. T. Lindenmeyer, W. Ju, C. S. Greene, B. Godfrey, C. D. Cohen, J. Krischer, M. Kretzler \& P. A. Merkel (2018) Metabolic pathways and immunometabolism in rare kidney diseases. Ann Rheum Dis, 77, 1226-1233.

Hua, W., H. Z. Huang, L. T. Tan, J. M. Wan, H. B. Gui, L. Zhao, X. Z. Ruan, X. M. Chen \& X. G. Du (2015) CD36 Mediated Fatty Acid-Induced Podocyte Apoptosis via Oxidative Stress. PLoS One, 10, e0127507.

Huggett, S. B. \& M. C. Stallings (2019) Cocaine'omics: Genome-wide and transcriptome-wide analyses provide biological insight into cocaine use and dependence. Addict Biol.

Jardim-Perassi, B. V., P. A. Alexandre, N. M. Sonehara, R. de Paula-Junior, O. Reis Junior, H. Fukumasu, R. Chammas, L. L. Coutinho \& D. Zuccari (2019) RNA-Seq transcriptome analysis shows anti-tumor actions of melatonin in a breast cancer xenograft model. Sci Rep, 9, 966.

Kennedy, D. J., Y. Chen, W. Huang, J. Viterna, J. Liu, K. Westfall, J. Tian, D. J. Bartlett, W. H. Tang, Z. Xie, J. I. Shapiro \& R. L. Silverstein (2013) CD36 and Na/K-ATPase-alpha1 form a proinflammatory signaling loop in kidney. Hypertension, 61, 216-24.

Langfelder, P. \& S. Horvath (2008) WGCNA: an R package for weighted correlation network analysis. BMC Bioinformatics, 9, 559.

Lin, Y. C., M. S. Wu, Y. F. Lin, C. R. Chen, C. Y. Chen, C. J. Chen, C. C. Shen, K. C. Chen \& C. C. Peng (2019) Nifedipine Modulates Renal Lipogenesis via the AMPK-SREBP

Transcriptional Pathway. Int J Mol Sci, 20.

Peer) reviewing PDF | (2019:07:39216:2:0:NEW 20 Aug 2019) 
393 Morrow, J. D., W. Qiu, D. Chhabra, S. I. Rennard, P. Belloni, A. Belousov, S. G. Pillai \& C. P.

394

395

396

397

398

399

400

401

402

403

404

405

406

407

408

409

410

411

412

413

414

415

416

417

418

419

420

421

422

423

424

425

426

427

428

429

430

431

Hersh (2015) Identifying a gene expression signature of frequent COPD exacerbations in peripheral blood using network methods. BMC Med Genomics, 8, 1.

Nosadini, R. \& G. Tonolo (2011) Role of oxidized low density lipoproteins and free fatty acids in the pathogenesis of glomerulopathy and tubulointerstitial lesions in type 2 diabetes. Nutr Metab Cardiovasc Dis, 21, 79-85.

Panousis, N. I., G. K. Bertsias, H. Ongen, I. Gergianaki, M. G. Tektonidou, M. Trachana, L. Romano-Palumbo, D. Bielser, C. Howald, C. Pamfil, A. Fanouriakis, D. Kosmara, A. Repa, P. Sidiropoulos, E. T. Dermitzakis \& D. T. Boumpas (2019) Combined genetic and transcriptome analysis of patients with SLE: distinct, targetable signatures for susceptibility and severity. Ann Rheum Dis, 78, 1079-1089.

Rees, F., M. Doherty, M. J. Grainge, P. Lanyon \& W. Zhang (2017) The worldwide incidence and prevalence of systemic lupus erythematosus: a systematic review of epidemiological studies. Rheumatology (Oxford), 56, 1945-1961.

Ritchie, M. E., B. Phipson, D. Wu, Y. Hu, C. W. Law, W. Shi \& G. K. Smyth (2015) limma powers differential expression analyses for RNA-sequencing and microarray studies. Nucleic Acids Res, 43, e47.

Ruan, X. Z., Z. Varghese, S. H. Powis \& J. F. Moorhead (1999) Human mesangial cells express inducible macrophage scavenger receptor. Kidney Int, 56, 440-51.

Shannon, P., A. Markiel, O. Ozier, N. S. Baliga, J. T. Wang, D. Ramage, N. Amin, B. Schwikowski \& T. Ideker (2003) Cytoscape: a software environment for integrated models of biomolecular interaction networks. Genome Res, 13, 2498-504.

Sun, G., P. Zhu, Y. Dai \& W. Chen (2019) Bioinformatics Analysis of the Core Genes Related to Lupus Nephritis Through a Network and Pathway-Based Approach. DNA Cell Biol, 38, 639650.

Tektonidou, M. G., A. Dasgupta \& M. M. Ward (2016) Risk of End-Stage Renal Disease in Patients With Lupus Nephritis, 1971-2015: A Systematic Review and Bayesian Meta-Analysis. Arthritis Rheumatol, 68, 1432-41.

Tsuboi, N., K. Asano, M. Lauterbach \& T. N. Mayadas (2008) Human neutrophil Fcgamma receptors initiate and play specialized nonredundant roles in antibody-mediated inflammatory diseases. Immunity, 28, 833-46.

van Dam, S., U. Vosa, A. van der Graaf, L. Franke \& J. P. de Magalhaes (2018) Gene coexpression analysis for functional classification and gene-disease predictions. Brief Bioinform, $19,575-592$.

Weening, J. J., V. D. D'Agati, M. M. Schwartz, S. V. Seshan, C. E. Alpers, G. B. Appel, J. E. Balow, J. A. Bruijn, T. Cook, F. Ferrario, A. B. Fogo, E. M. Ginzler, L. Hebert, G. Hill, P. Hill, J. C. Jennette, N. C. Kong, P. Lesavre, M. Lockshin, L. M. Looi, H. Makino, L. A. Moura \& M. Nagata (2004) The classification of glomerulonephritis in systemic lupus erythematosus revisited. J Am Soc Nephrol, 15, 241-50.

Peer) reviewing PDF | (2019:07:39216:2:0:NEW 20 Aug 2019) 
432 Weening, J. J., V. D. D'Agati, M. M. Schwartz, S. V. Seshan, C. E. Alpers, G. B. Appel, J. E. 433 Balow, J. A. Bruijn, T. Cook, F. Ferrario, A. B. Fogo, E. M. Ginzler, L. Hebert, G. Hill, P. Hill, 434 J. C. Jennette, N. C. Kong, P. Lesavre, M. Lockshin, L. M. Looi, H. Makino, L. A. Moura \& M. 435 Nagata (2004) The classification of glomerulonephritis in systemic lupus erythematosus 436 revisited. J Am Soc Nephrol, 15, 241-50.

437 Yang, X., D. M. Okamura, X. Lu, Y. Chen, J. Moorhead, Z. Varghese \& X. Z. Ruan (2017) 438 CD36 in chronic kidney disease: novel insights and therapeutic opportunities. Nat Rev Nephrol, 439 13, 769-781.

440 Yang, X., Y. Wu, Q. Li, G. Zhang, M. Wang, H. Yang \& Q. Li (2018) CD36 Promotes Podocyte 441 Apoptosis by Activating the Pyrin Domain-Containing-3 (NLRP3) Inflammasome in Primary 442 Nephrotic Syndrome. Med Sci Monit, 24, 6832-6839.

443 Yu, G., L. G. Wang, Y. Han \& Q. Y. He (2012) clusterProfiler: an R package for comparing 444 biological themes among gene clusters. Omics, 16, 284-7. 
Figure 1

Flow chart of the whole procedures in this study.

Data processing, analyses, hub gene identification and validation.

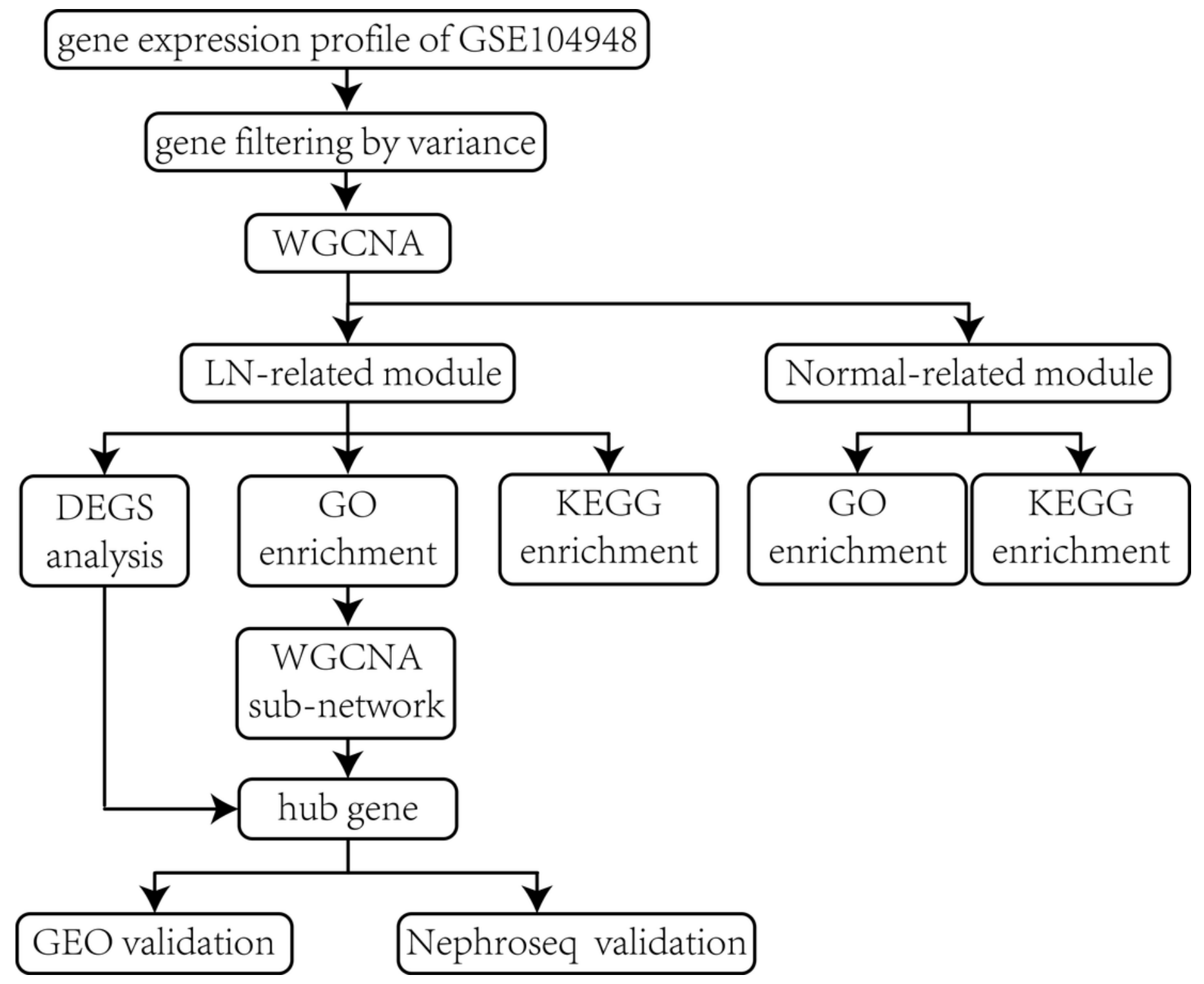




\section{Figure 2}

Sample cluster dendrogram and soft-thresholding values $(\beta)$ estimation.

(A) Sample cluster dendrogram and clinical trait heatmap of 18 normal samples and 21 LN samples based on their expression profile. (B) Analysis of scale-free fit index of each $\beta$ value from 1 to 30. (C) Analysis of mean connectivity of each $\beta$ value from 1 to $30 . \beta=10$ was chosen for subsequent analyses as it has the biggest mean connectivity when the scale-free fit index is up to 0.85 . 
A

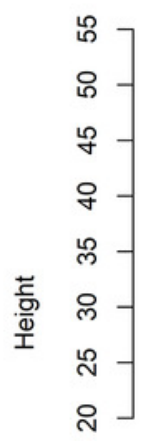

B

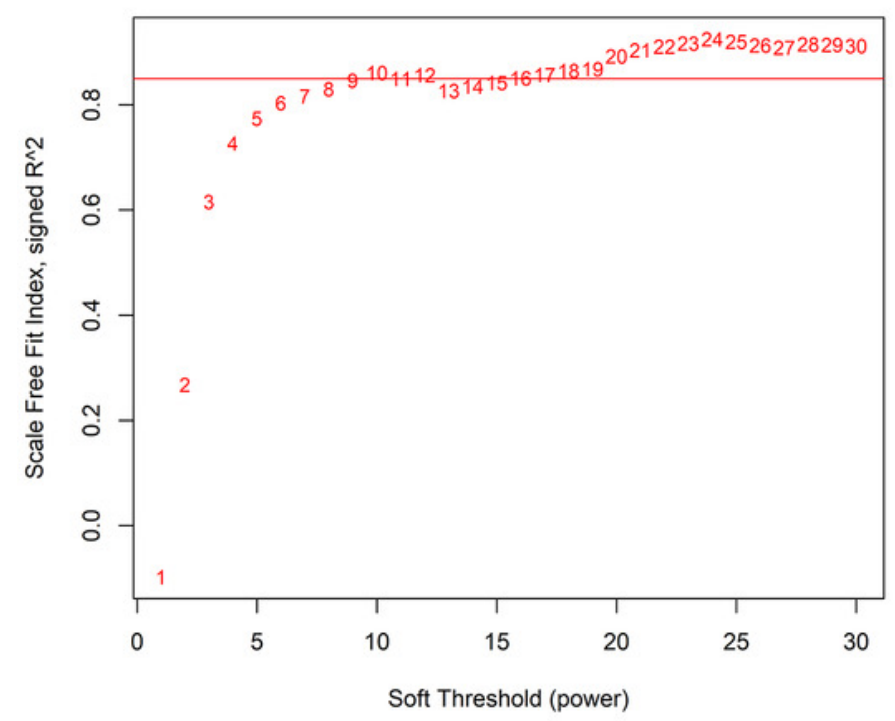

Sample dendrogram and trait heatmap

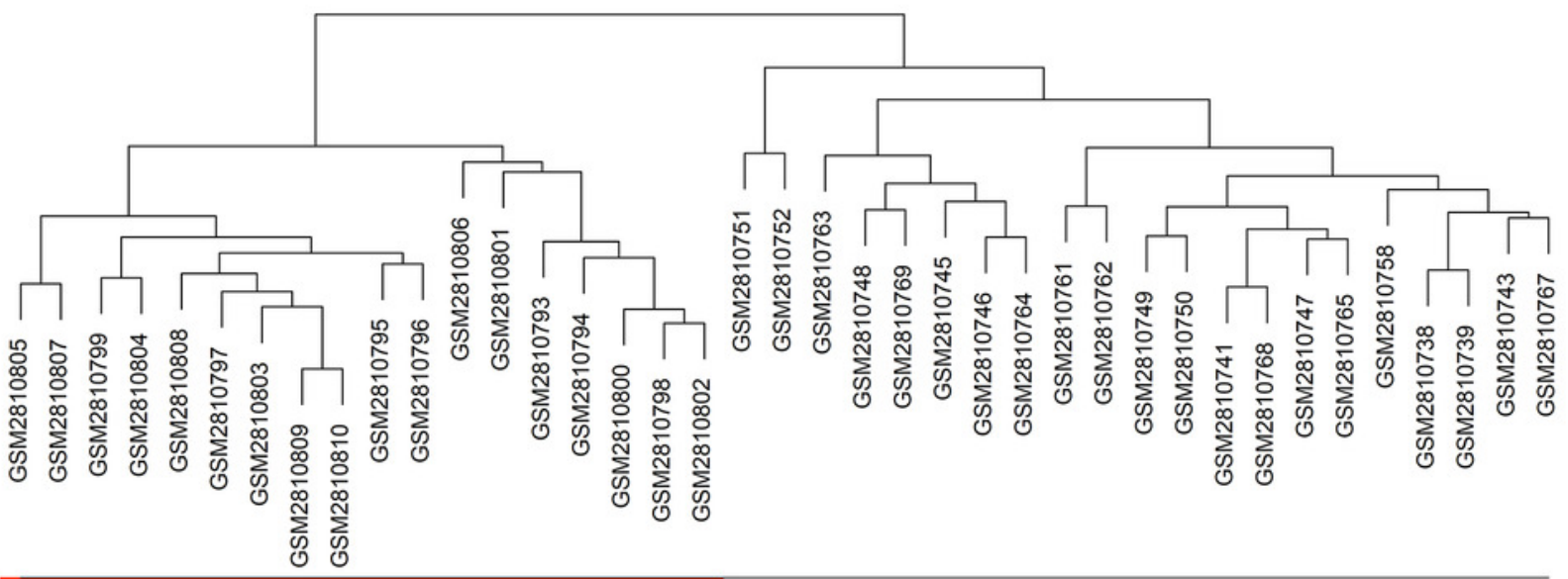

NORMAL

LN

$\mathrm{C}$

Mean connectivity

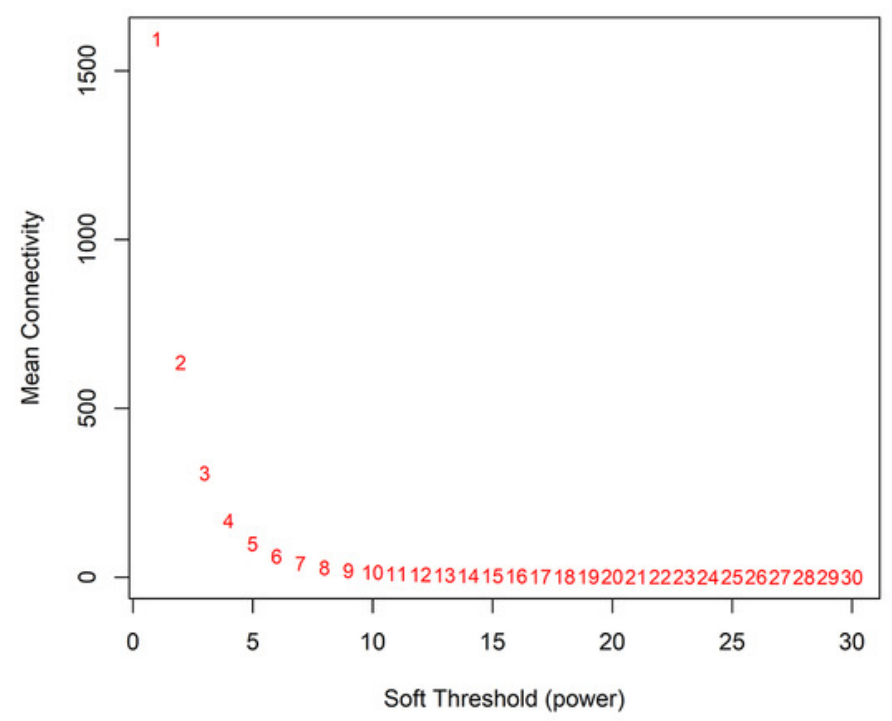




\section{Figure 3}

Division and validation of co-expression modules.

(A) Dendrogram of all genes divided into 6 modules base on a dissimilarity measure (1-TOM). The modules labeled by color are indicated below the dendrogram. The upper presents the original division by average linkage hierarchical clustering according to the TOM-based dissimilarity measure and the under presents the modules merged according to the Pearson's correlation of eigengenes. (B) Adjacency heatmap of the 5,942 genes analyzed by WGCNA. The depth of the red color indicates the correlation between all pair-wise genes. The red color mainly distributes in the diagonal of the heatmap. (C) Clustering dendrogram of eigengenes. (D) Adjacency heatmap of eigengenes. Red represented high adjacency (positive correlation) and blue represented low adjacency (negative correlation). 


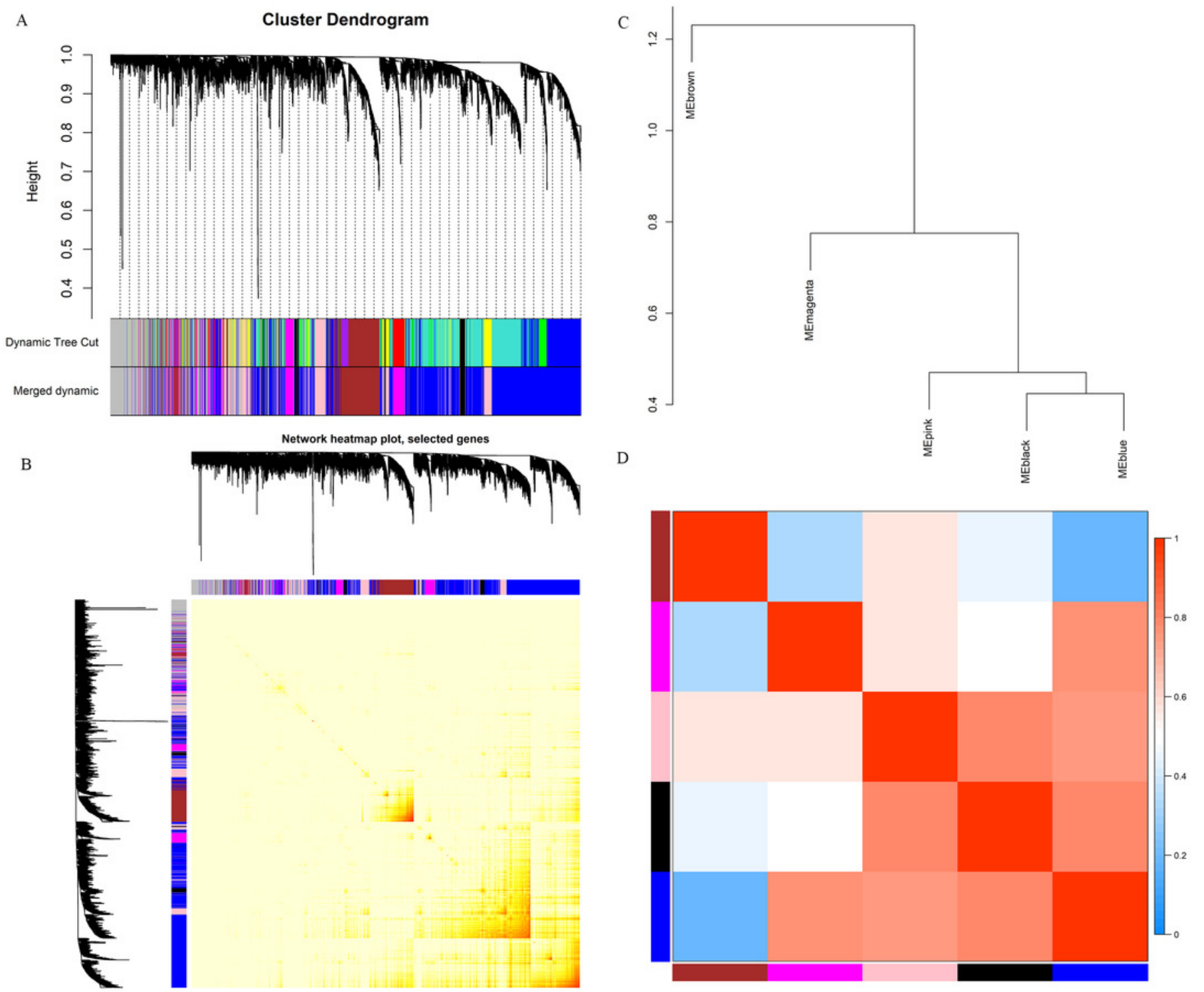




\section{Figure 4}

Identification and verification of clinical related modules.

(A) Heatmap of module-trait correlations. Each cell depicts the correlation coefficients and $P$ value. The cells are colored by the intensity of correlation according to the color legend (red for positive correlation and blue for negative correlation). The blue module (top LN module) and brown module (top non-LN module) were identified as trait-related modules. (B) Scatter plot for correlation between the Gene significance (GS) and Module Membership (MM) in the top LN module. Correlation coefficients and P-value is labeled at the top. (C) Scatter plot for correlation between the GS and MM in the top non-LN module. 
A

Module-trait relationships

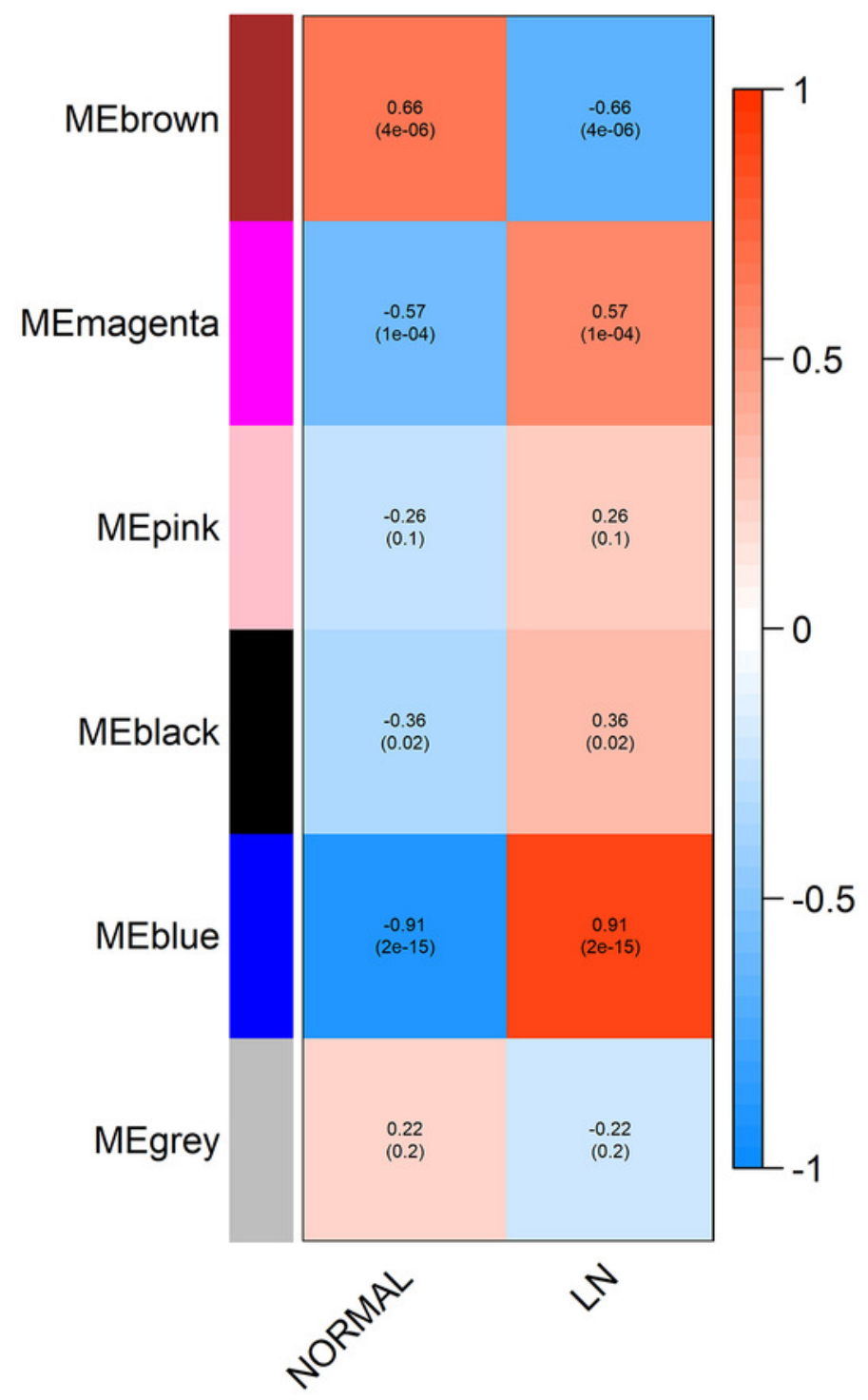

B Module membership vs. gene significance cor $=0.89, p<1 e-200$

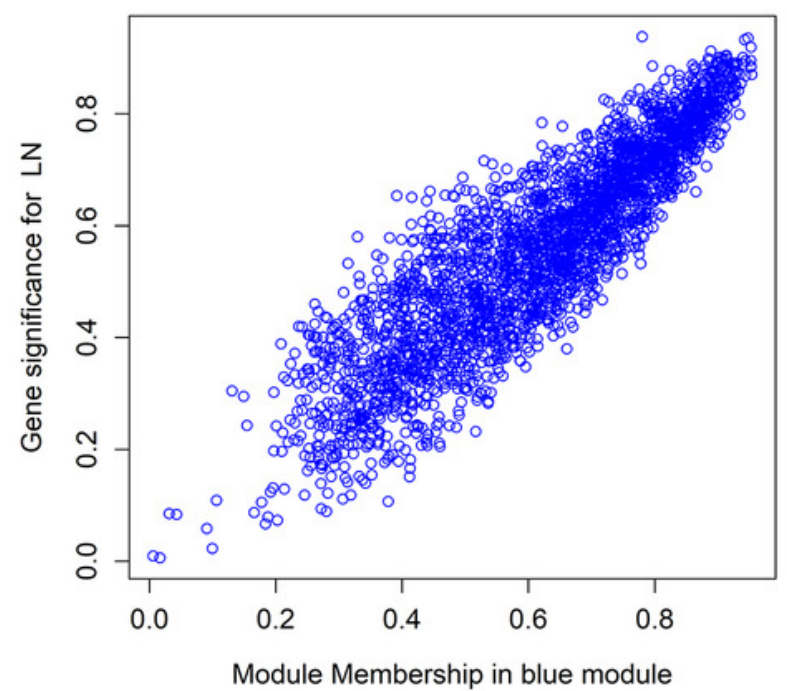

C Module membership vs. gene significance cor $=0.73, p=3.1 e-130$

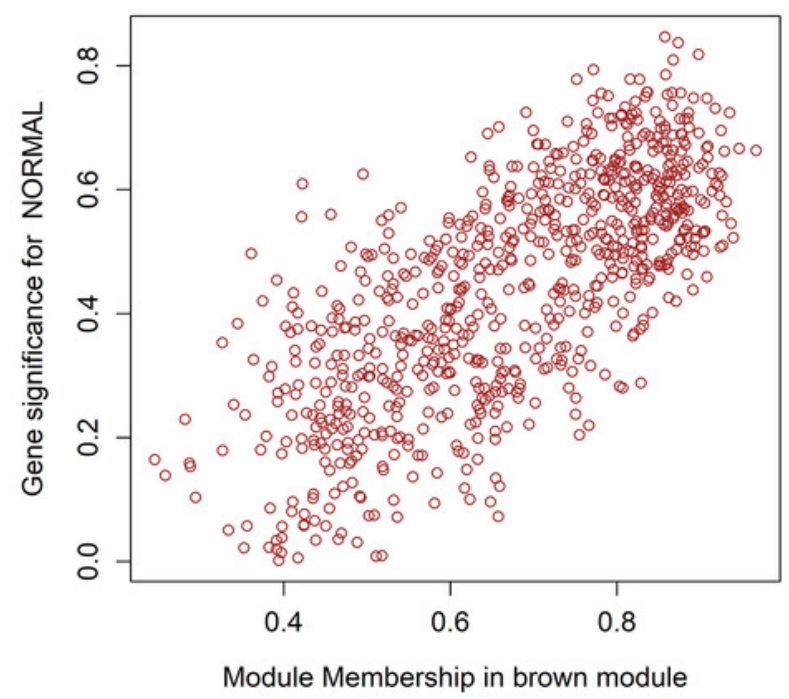




\section{Figure 5}

DEGs analysis of two trait-related modules.

The color of each cell represents the expression level of a gene in a sample (red for high level and blue for low level). Only DEGs with top $30 \log F C$ values are displayed. (A) Heatmap of DEGs in the top LN module. (B) Heatmap of DEGs in the top non-LN module.
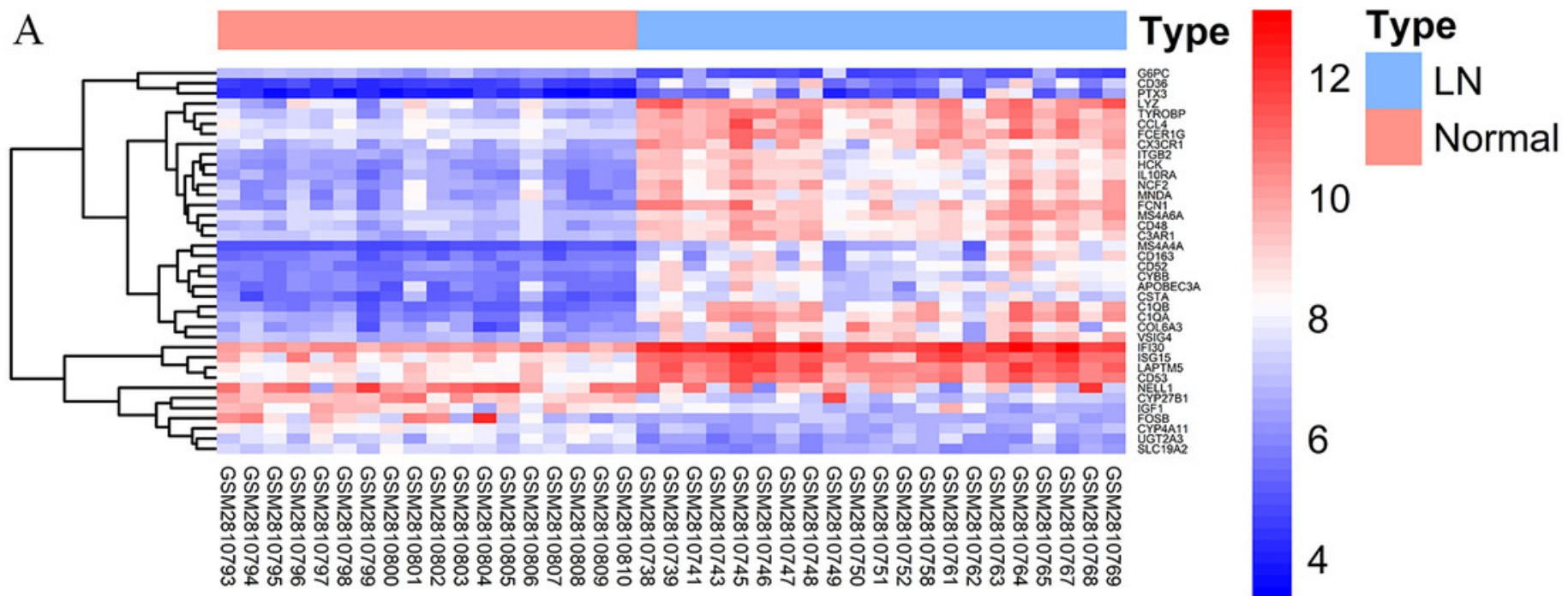

10

8

6

4
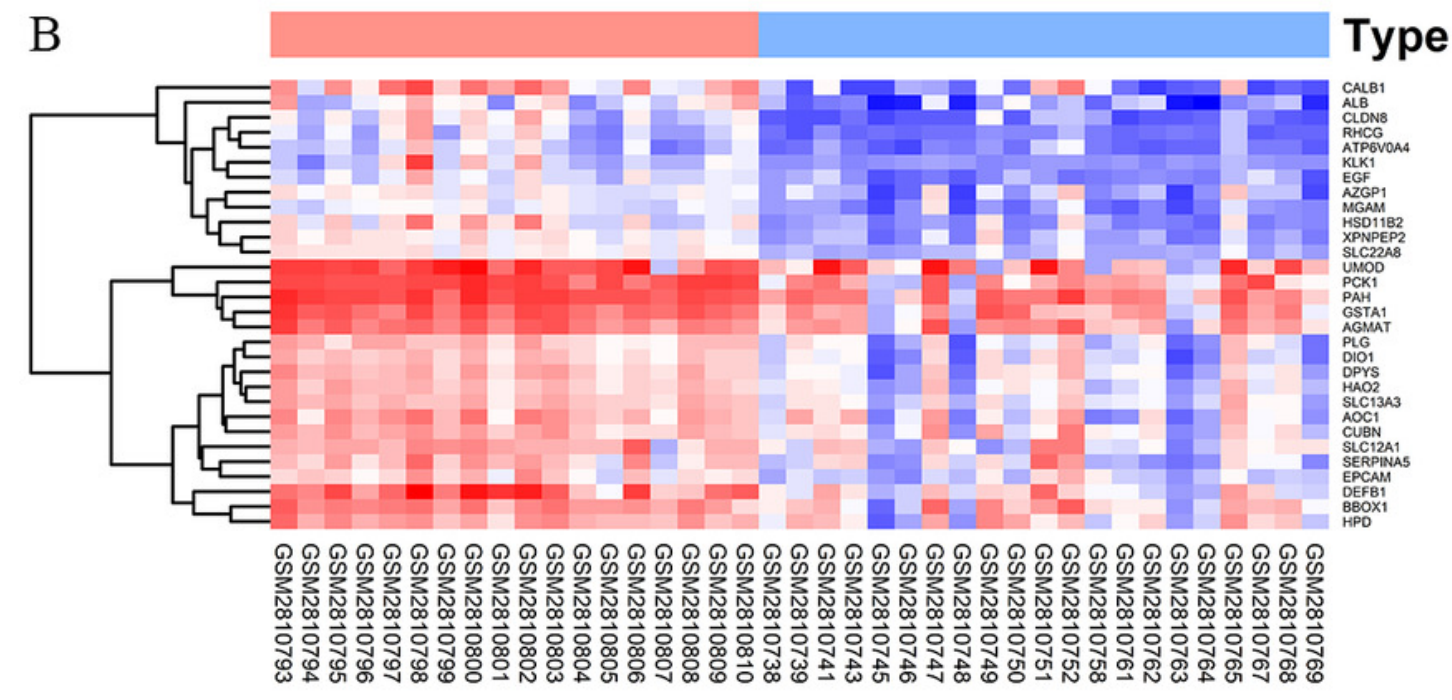

CALB1
ALB
CLONB
RHNB

CLB
CLNS
RHCG
ATPGVOA

APRGVOA
KLIK1
EGF
EF

EGF
AZPA1
MGAM

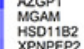

XPNPEP 2
SSC2AB
UMOOD

PCK1
PAH
GSAA1

AGMAT
PLG
DIO1

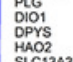

HAO2
SLC13A3
AOC1
CUBN

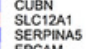

EPCAA
EEFB!
DEF

\section{P}

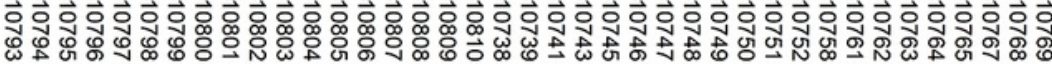

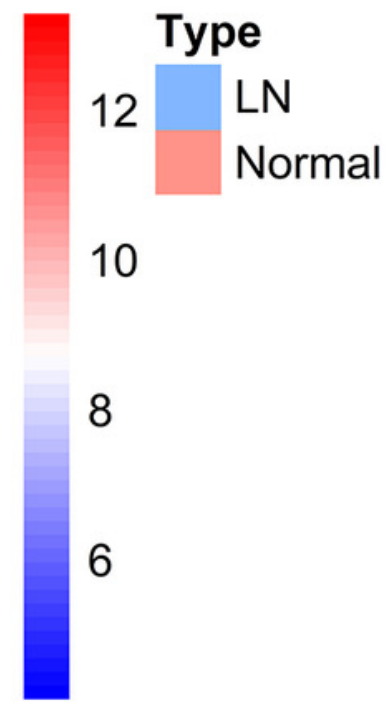




\section{Figure 6}

GO enrichment analyses of two trait-related modules.

The depth of color is corresponded to the enrichment significant of each term and the $x$-axis indicates the enriched gene count. (A) Top 10 significantly enriched GO Biological Process (BP) terms of top LN module. (B) Top 10 significantly enriched GO Molecular Function (MF) terms of top LN module. (C) Top 10 significantly enriched GO Cellular Component (CC) terms of top LN module. (D) Top 10 significantly enriched GO Biological Process (BP) terms of top non-LN module. (E) Top 10 significantly enriched GO Molecular Function (MF) terms of top non-LN module. (F) Top 10 significantly enriched GO Cellular Component (CC) terms of top non-LN module. 


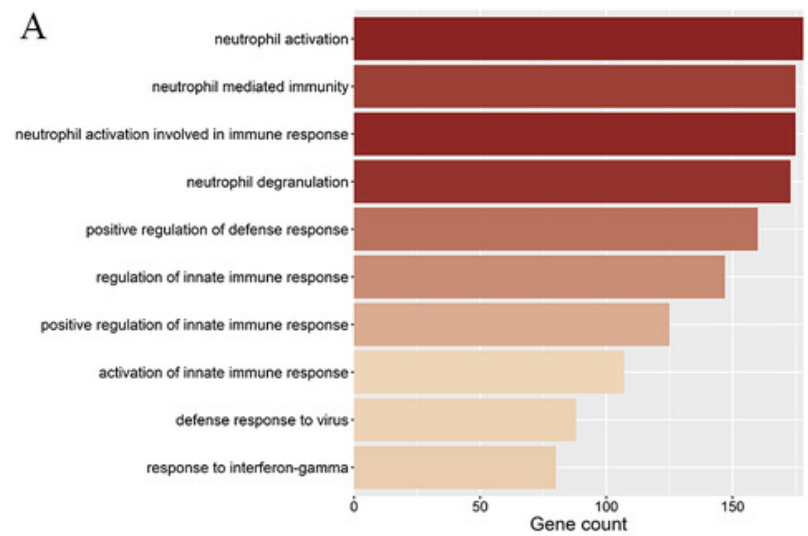

B

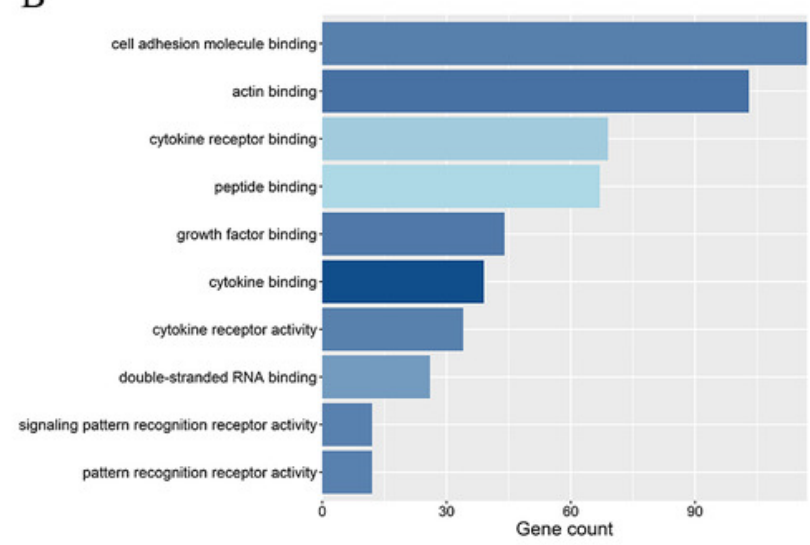

C

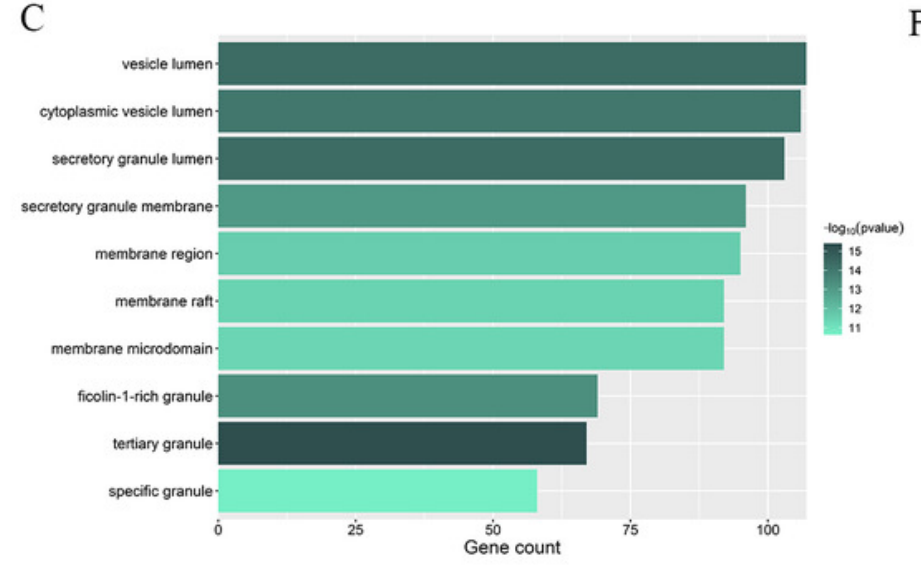

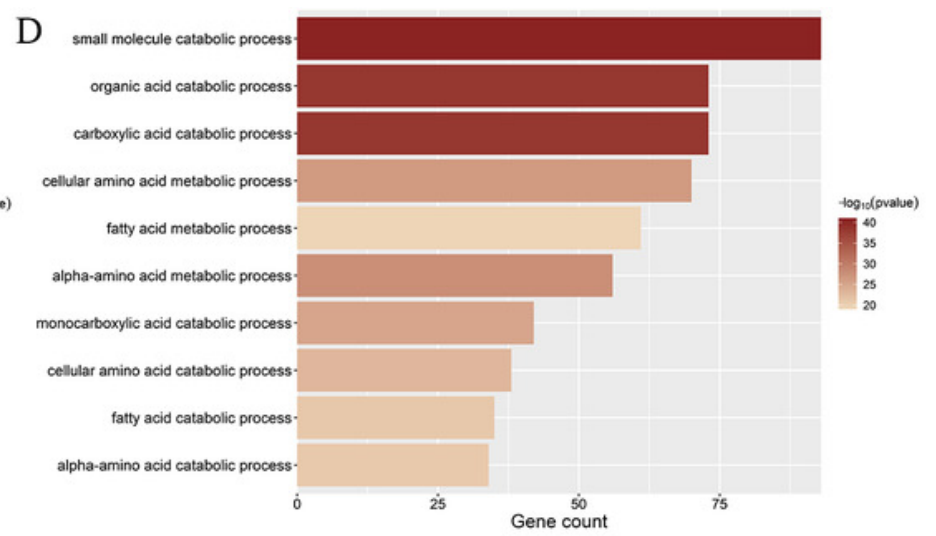

E

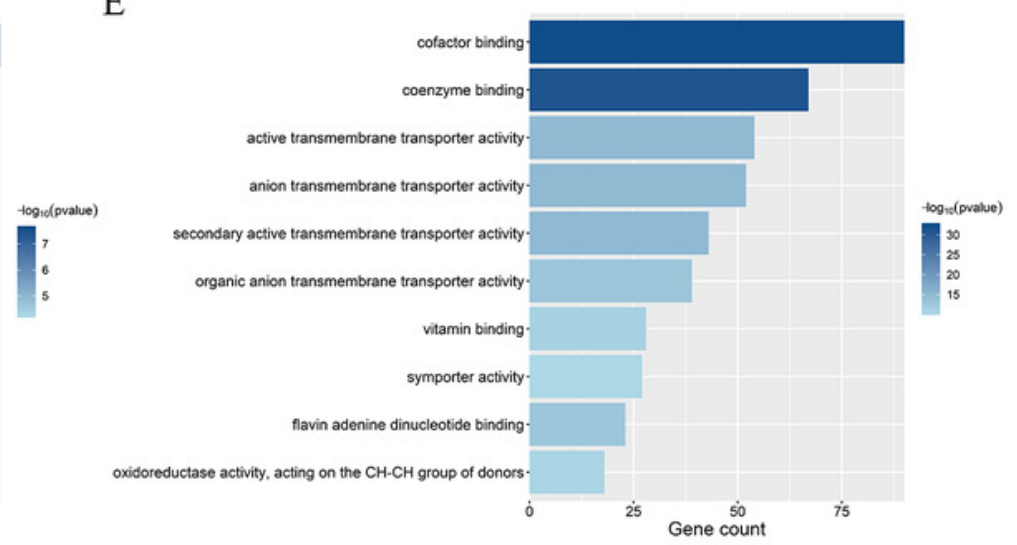

F

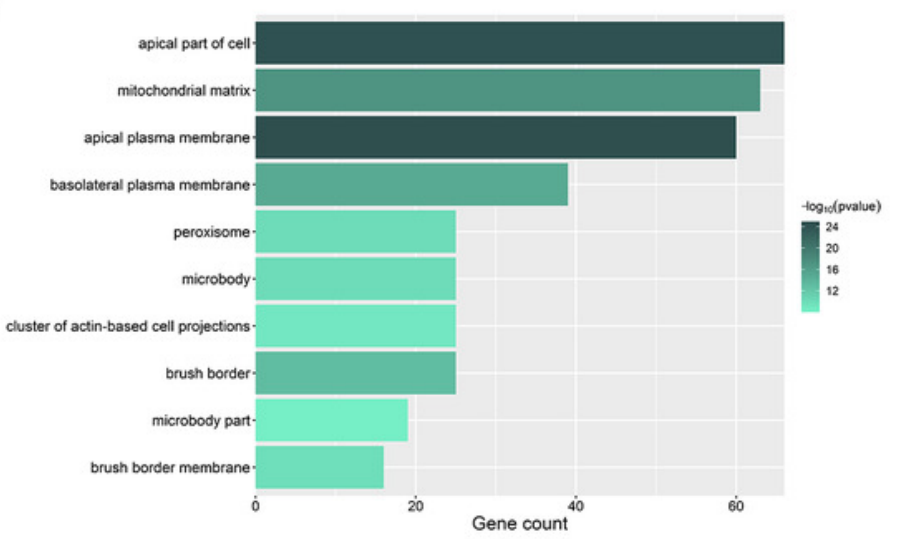




\section{Figure 7}

KEGG enrichment analyses of two trait-related modules.

The depth of color is corresponded to the enrichment significant of each term and the size of the circle indicates the enriched gene count. (A) Top 10 significantly enriched KEGG terms of top LN module. (B) Top 10 significantly enriched KEGG terms of top non-LN module. 


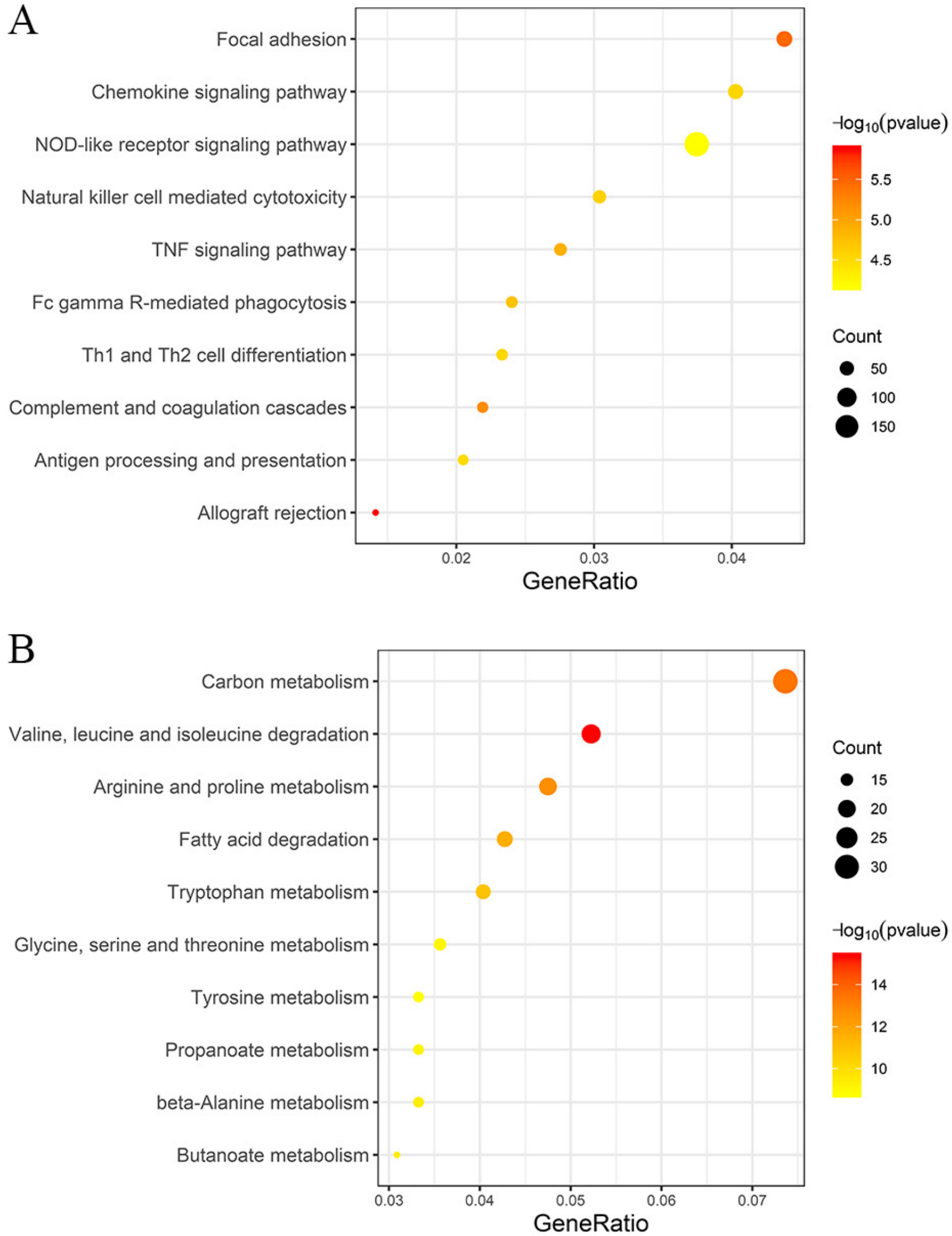




\section{Figure 8}

Sub-networks of WGCNA based extracted based on most significant GO terms.

The nodes represent the genes and the edges represent the weighted correlation. Only coexpression pairs with top 500 weighted correlations are included. The red and yellow nodes represent genes of top 10 MCC values (red for a higher MCC value and yellow for lower). (A) Sub-network of the GO term "neutrophil activation". (B) Sub-network of the GO term "positive regulation of defense response".
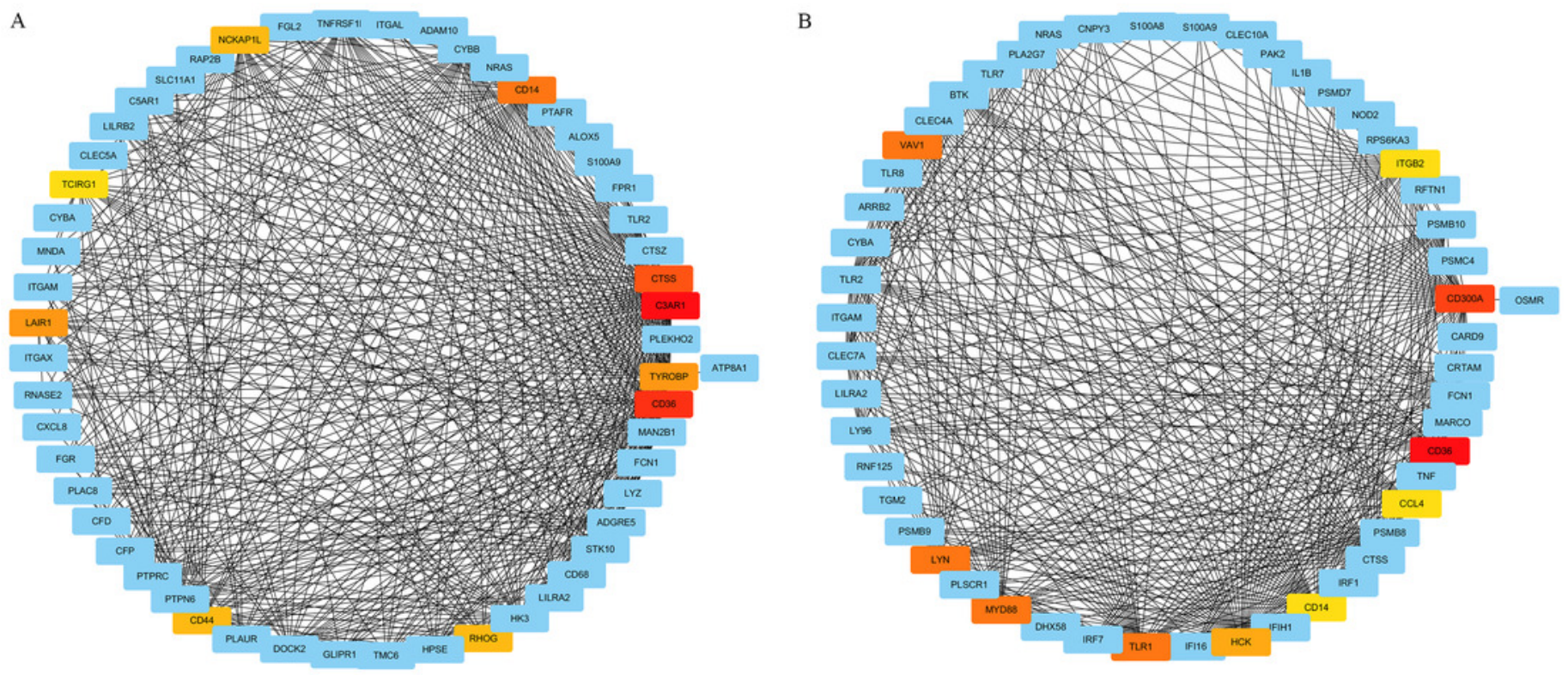
Figure 9

Differentially expressed level of CD36 between LN and normal in different GEO datasets.

(A) Expression level of CD36 in dataset of GSE104948. (B) Expression level of CD36 in dataset of GSE32591. (C) Expression level of CD36 in dataset of GSE99339. (D) Expression level of CD36 in dataset of GSE113342.

A

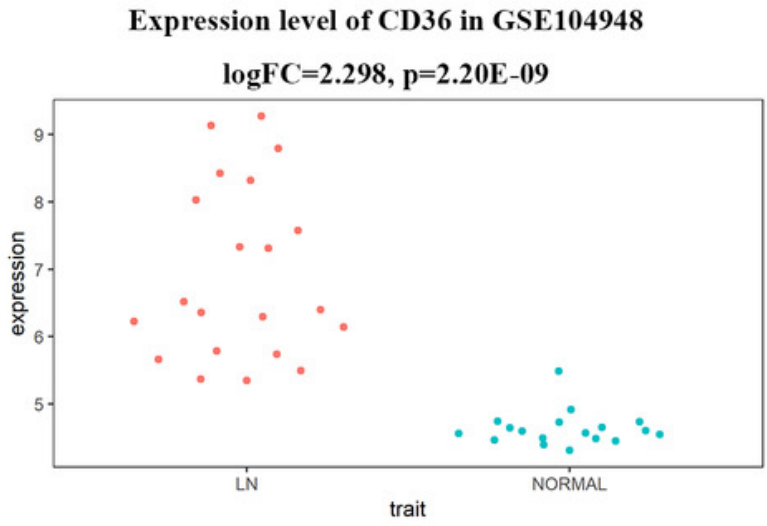

B

Expression level of CD 36 in GSE32591

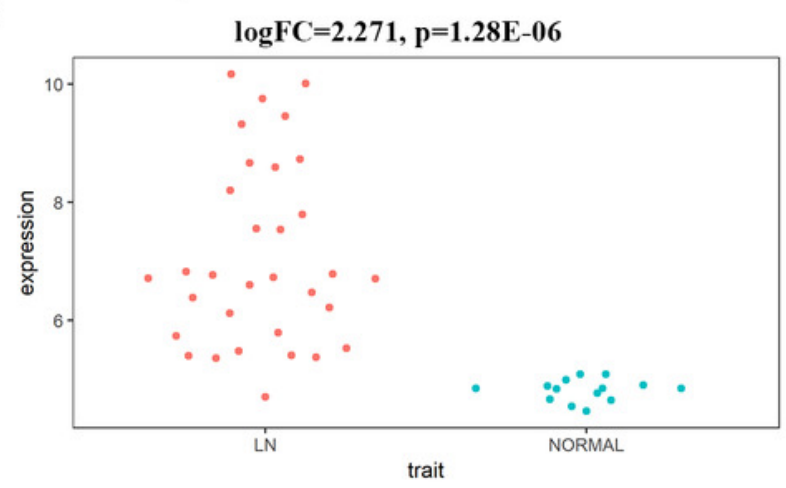

$\mathrm{C}$

Expression level of CD36 in GSE99339

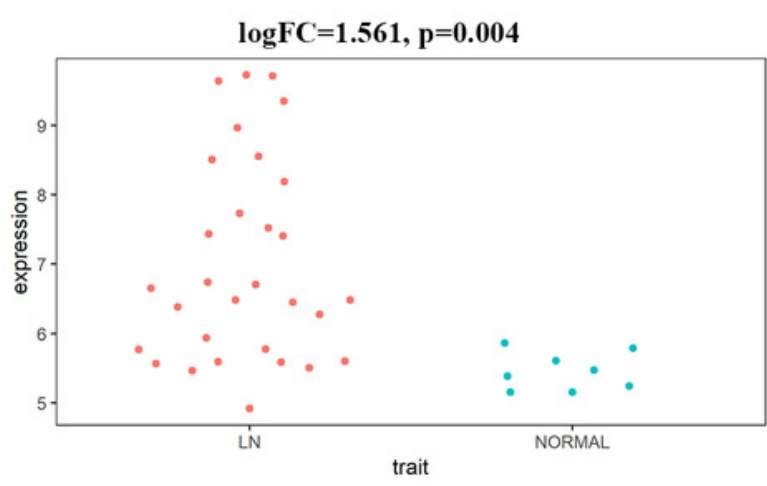

Expression level of CD36 in GSE113342

$\mathrm{D}$

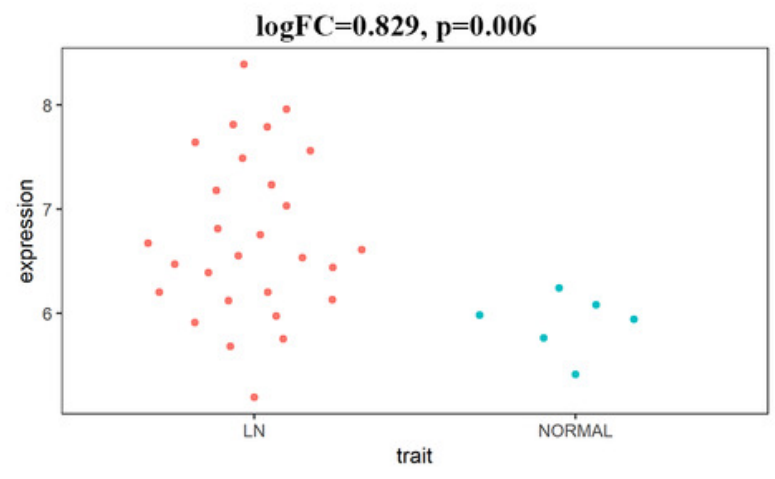


Figure 10

Differentially expressed level of CD36 in glomerular tissues of different WHO Lupus Nephritis Class.

(A) Differentially expressed level of CD36 in class II and class IV respectively (from the dataset of Berthier Lupus Glom). (B) Differentially expressed level of CD36 in class III and class IV respectively (from the dataset of Peterson Lupus Glom).

A Expression of CD36 in different WHO Lupus Nephritis Class

Fold change $=2.603, \mathrm{p}=0.026$

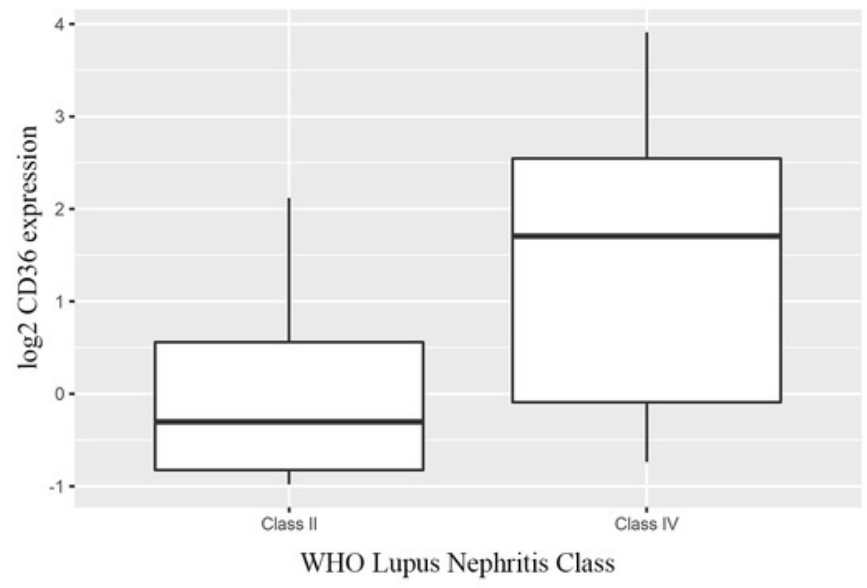

B Expression of CD36 in different WHO Lupus Nephritis Class Fold change $=1.848, \mathrm{p}=4.61 \mathrm{e}-5$

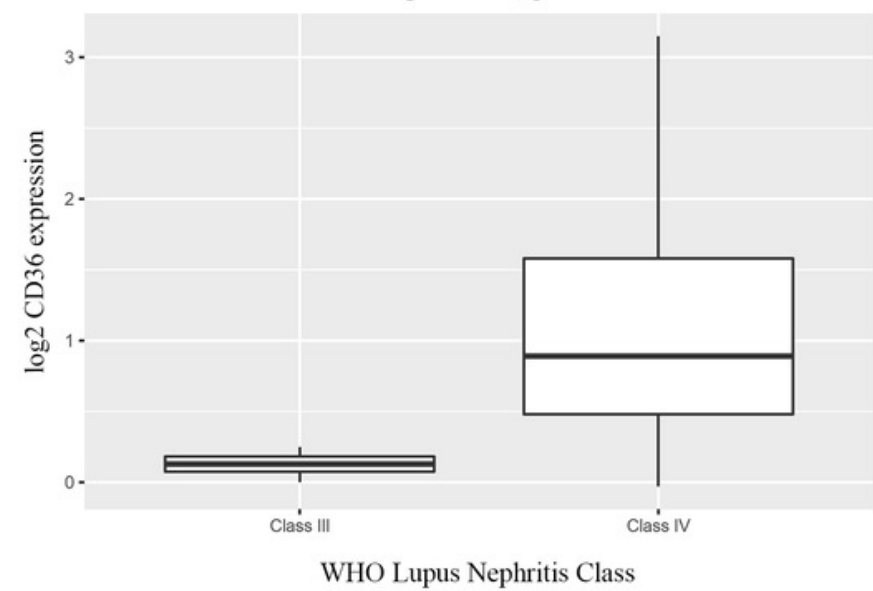

